\title{
Knowledge Practices Environment: Design and Application of Technology for Trialogical Learning
}

\author{
Patrick Sins $^{1}$, Merja Bauters ${ }^{2}$ and Crina Damşa ${ }^{1}$ \\ ${ }^{1}$ Research Centre Learning in Interaction, Utrecht University, PO Box 80410, \\ 3508 TC Utrecht, The Netherlands \\ \{P.H.M.Sins, C.I.Damsa\}@uu.nl \\ ${ }^{2}$ EVTEK University of Applied Sciences, Vanha Maantie 6, FI-02650 Espoo, Finland \\ Merja.Bauters@evtek.fi
}

\begin{abstract}
Current networked society present learners with challenges that cannot be sufficiently coped with in educational contexts that are characterized by transmission or participation epistemologies on learning. To address these challenges, the trialogical approach to learning is introduced which focuses on the ways in which people and their communities create knowledge through the tool-mediated development of shared objects. Supporting sustained collaborative knowledge creation requires learning technology that is modular and extensible rather than monolithic and fixed in nature which characterizes most existent learning technologies. The current paper presents the design considerations and the application of the KP-environment which aims to support these object-oriented practices.
\end{abstract}

Keywords: Knowledge Creation, Design of Learning Technology, Knowledge Management, Pedagogical Scenarios

\section{Introduction}

Rapid changes in current knowledge society present new challenges to human competencies. Productive participation in knowledge-intensive work requires that individuals, their professional communities, and their organizations develop new skills, advance their knowledge and their understanding as well as produce innovations. This is reflected in developments in professional communities wherein work is increasingly focused on the deliberate advancement of knowledge rather than on the mere production of material objects [1]. In order to conceptualize and understand the nature of work and activity in current knowledge society, one has to comprehend the various types of knowledge that intersect within complex and heterogeneous networks which consist of humans and various artifacts [2, 3]. In parallel with these changes in society, conceptions on learning, knowledge practices, and social organization of learning also have to be transformed so as to facilitate corresponding competencies which enable student to cope with these emerging challenges.

Educational institutions that make an attempt at addressing these knowledge practices in their pedagogical approach, are challenged to redesign (aspects of) their 
curriculum as well as to develop the practices and professionalism of their educators. However, although we are in a period of change, educational practice still has many characteristics of the transmission scenario [4] on the one hand or on mere social and cultural interaction on the other. These scenarios, which corresponds to the premises of respectively the acquisition and participation metaphors to learning [5] and which characterizes most formal education, centers on the acquisition of declarative knowledge and a limited number of critical skills, by a system of lectures, textbooks, and testing. As a consequence to cope with the cognitive, social, and motivational challenges of the emerging knowledge-based society, technological tools and educational methods are needed to improve the quality of learning and to transform the educational system accordingly.

Paavola \& Hakkarainen's model of knowledge-creation [6] provides a framework to support educators to develop and advance their practices of learning and instruction. A central feature in the knowledge-creation approach is mediation [7]; meaning that people collaboratively create knowledge through the development and advancement of shared objects. It is characteristic of this kind of knowledge advancement that it takes place within innovative knowledge communities which are organized around shared objects whose creation and development defines their purpose [8]. The knowledge-creation view represents a 'trialogical' approach because the emphasis is not only on individuals or on community, but on the way people collaboratively develop epistemic artifacts.

Current learning technologies provide only limited support for these kinds of collaborative practices, but instead serve as repositories for knowledge objects or as virtual collaboration platforms instead.

The purpose of the present paper is to illuminate the design considerations and the application of technology which aims to support trialogical learning: the Knowledge Practices environment (KP-Environment). The KP-Environment is developed from within the frame of the Knowledge Practices Laboratory project, a 5 year IST project co-funded by the European Community.

The next section present an overview of the challenges involved in the design of the KP-environment. Subsequently, we describe its basic architecture and demonstrate how the KP-environment can support authentic trialogical practices employing a pedagogical scenario. Finally, we conclude with the ways in which empirical research will feed into the iterative re-design of the KP-environment.

\section{Designing Technological Support for Trialogical Learning}

The current approaches of working with knowledge in educational and workplace settings, however, are still focused on individuals' skills and knowledge structures on the one hand, or on social and cultural interaction on the other hand. The problem is that they do not provide sufficient models for facilitating processes of knowledge creation with related practical, organizational and technological means. As a consequence there is a lack of pedagogically and scientifically sound technological tools to foster competencies for knowledge creation among students and professionals alike. 
Technological support for trialogical learning requires software tools that support spatially, socially and temporally distributed participation in trialogical processes and draw on the potential of emerging semantic web technologies. This involves mainly the need for a modular, flexible, and extensible ICT system that supports trialogical learning in educational and workplace settings. In specific, this means that in tools should be able to address collaborative work around shared objects and to support knowledge practices in various educational settings.

Current learning technologies such as the Moodle platform or Blackboard provide only limited support for collaborative knowledge practices. These tools typically provide support for information sharing and for participation in social interaction. The few existing eLearning applications that support specific models of knowledge creation processes such as the Future Learning Environment (FLE) or Knowledge Forum are of limited use because of their inflexible and monolithic software design. While many core technologies of the Semantic Web infrastructure are already available, there is vast amount of work ahead in making them more usable for learners and their instructors.

Learning technologies and knowledge management systems have each received a significant interest in research and development within the past two decades. However, they have been remarkably separated by their context specific applications and few linkages exploiting their synergies have been established. Software for managing knowledge processes is another poorly developed area. The workflow applications are designed to support structured business processes and do not provide support for dynamically evolving knowledge creation processes. There are some initiatives related to computer supported collaborative learning that have developed software to support specific models of knowledge creation processes. However, these tend to focus on one aspect of knowledge creation processes such as the "rise above" focus in Knowledge Forum.

One central way of developing new methodological solutions in the design of technology for the learning sciences is the use of design principles to guide the design of educational practices and technology. Various ways of developing design principles have been suggested, both top-down, i.e. theory driven [9], and bottom-up, i.e. empirically informed [10] approaches. A key challenge in the design of KPenvironment is to capitalize on both theory as well as insights gained from educational practice to serve as guidance in the development of tools.

\section{Design Principles for Pedagogical Scenarios of Trialogical Learning}

Design of KP-environment was based upon design principles which are grounded in the perspective of trialogical approach on learning [6]. The design principles serve as guide and as generic criteria in the design process in the sense that they enable the creation of pedagogical scenarios which attempt to situate and contextualize the knowledge practices within particular educational contexts. Hence, these scenarios can be conceptualized as the concretization of the design principles for designing technology supporting trialogical learning. 
Nevertheless, the design principles are provisional implying that they reflect the current understanding of trialogical approach to learning and will therefore be subject to continuously review and if necessary revision or extension based on empirical results. The design principles that were taken into consideration in the design of KPenvironment were:

1. Focus on trialogical activity around shared objects: A central idea of the trialogical approach to learning is that work and learning are organized around developing some shared objects. Tools should facilitate work and learning around shared objects of activity, not just individual, cognitive processing nor social interaction as such;

2. Interaction between personal and social level: The aim is to develop tools, pedagogical models and spaces for combining developed social practices with room for individual initiatives for developing shared objects.

3. Fostering long-term processes of knowledge advancement: Processes of transforming existing knowledge practices and creating new knowledge are mostly longitudinal in nature. Tools and practices should be provided which support knowledge practices and collaboration around shared objects not just here-and-now but which moreover facilitate sustained knowledge creation;

4. Development through transformation and reflection: Models and theories belonging to the knowledge creation approach emphasize development through interaction between various forms of knowledge and between practices and conceptualizations which is the driving force in the process of knowledge creation. Tools and practices have foster such transformations;

5. Eliciting (individual and collective) agency: The trialogical approach to learning has its basis on epistemic agency of the participants; both agency of individual participants on their own efforts but also on collective agency supporting social processes and collaborative efforts. Therefore students have to engage themselves in planning, monitoring, and evaluating their collective and individual activities in order to develop higher-order competencies and knowledge practices;

6. Cross fertilization of knowledge practices: Tools and models have to be designed to assist people to solve complex, authentic problems and produce objects also for purposes outside educational institutions. It has its basis on cross fertilization of knowledge practices between various educational institutions (like polytechnics and universities), on the one hand, and between educational institutions and professional organizations, on the other hand;

7. Flexible tool mediation for trialogical activity: The trialogical approach has its basis on flexible tools which facilitate the integration between those aspects which are highlighted in other design principles, that is, long-term, cross fertilized work around shared objects which help an interaction between personal and social levels, and which support to make transformations between various forms of knowledge.

Scenarios can be employed to concretize the practices and pedagogical context in which the KP-environment is used and provide the background for the further design of the tool. Scenarios provide a mediating artifact to plan, implement and carry out a pedagogical or professional intervention and to describe the nature of the activities that are supported by the KP-environment.

A scenario that illustrates how these design principles of the trialogical approach to learning could be actualized in certain practices in higher education and that describes 
how the tools of KP-environment can support trialogical practices is the Bachelor Thesis scenario. This scenario is based on an existing third-year Bachelor course that is offered at the Department of Educational Sciences of Utrecht University, the Netherlands.

\subsection{Short Description of the Bachelor Thesis Scenario}

The main aim of this course is to support students to learn more about conducting research within the social sciences and to develop skills in collaborative academic writing. Students are required to collaboratively plan, conduct and report on a research project involving topics that are relevant within the field of education, supported by a team of educational scientists. The final product of each group is a collaboratively written scientific paper and a presentation of the paper during a Bachelor Thesis congress day.

Knowledge creation and knowledge advancement is reflected at the product as well as at the process level, while the pedagogical set-up offers opportunities for the team members to work together on shared epistemic artifacts (e.g. research plan, preliminary research report, final research report, and data collection instruments). Team efforts are required when making decisions, conducting analysis, processing data, when reporting on the collected data or brainstorming for constructing tools for analyzing the data. In addition, students are challenged to apply knowledge gained from previous courses in a new and more open-ended situation which requires them to create new understandings concerning issues related to conducting scientific research. Throughout the course the students are provided with web-based technological tools, which support their collaborative practices which are mediated by their objects of activity.

In the next section the KP-Environment will be presented in addition to how the Bachelor Thesis scenario can be applied to show how the design principles of trialogical learning are reflected in the ways the KP-environment can support students' knowledge practices.

\section{The Knowledge Practices Environment}

KP-environment is a virtual collaboration space offering facilities for interacting with knowledge artifacts, knowledge process models, users and the KP-environment itself during a trialogical learning or working process. KP-environment provides common tools for re-structuring and organizing knowledge artifacts based on their conceptual semantics. A KP-environment can be either both a personal space as well as a collective space. A collective space is created for the knowledge community involved in a trialogical process. The knowledge community can be formed around a group of people belonging to e.g. project team, students attending a class, or students of a university department, or any other type of collective. A KP-environment provides the user with a configurable set of tools for: 
- working with knowledge artifacts (e.g. creating, editing, storing, sharing, commenting, adding semantic descriptions or tags, disseminating and discussing);

- managing the knowledge processes (e.g. creating, changing and executing process models);

- managing the KP-environment itself (e.g. configuring the tools available).

At the level of the general graphical user interface (GUI) the KP-Environment consists of a set of shared spaces which involve collective workplaces for students to use. This means that in the KP-environment the different courses as well as all materials offered and produced are encapsulated in shared spaces as shown in Figure 1.

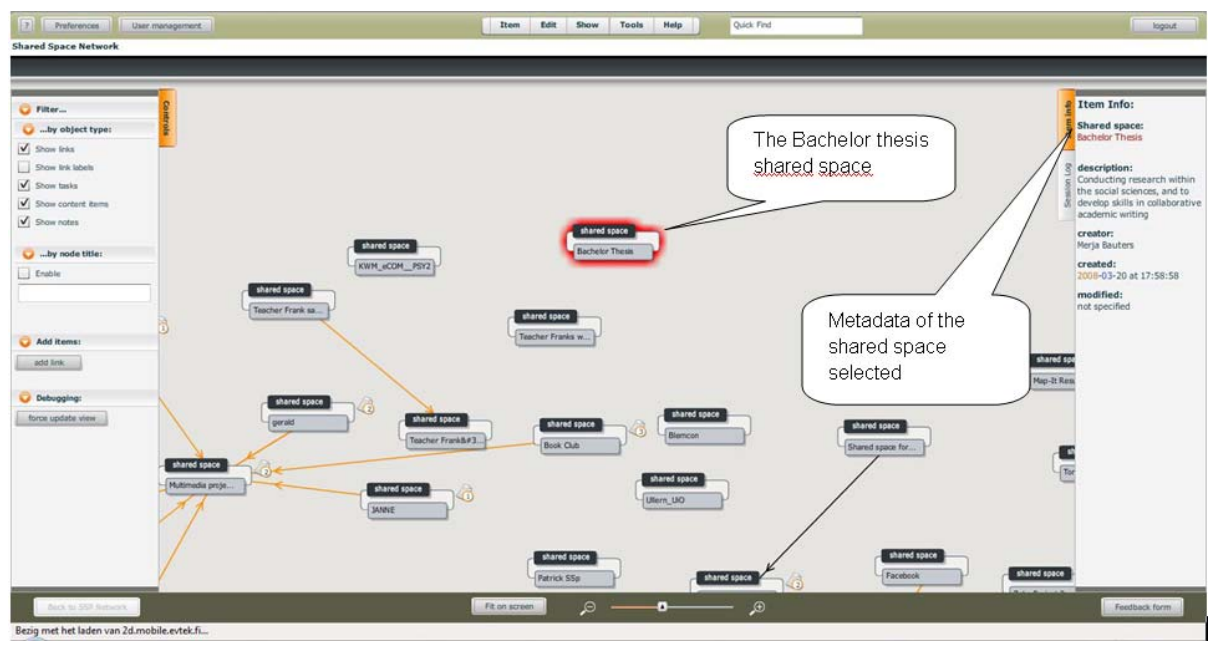

Fig. 1. Overview of shared spaces in the KP-Environment

Each shared space encompasses a workplace for students which presents them with three views: a Process view, a Content item view and a Community view. The process view presents students with a GANTT chart which specifies the tasks students have set for themselves, allocated responsibilities, artifacts to be produced and deadlines (see Figure 2). This functionality enables students to manage their knowledge creation processes themselves and fosters collective agency supporting social processes and collaborative efforts.

The Content item view presents students with the tasks (light gray), a graphical representation of all the artifacts produced in service of performing these tasks and their linkages and metadata describing these artifacts (see Figure 3). The content items (dark grey) represent the artifacts students' have produced but also contain the semantics and contents of that particular artifact. Content items can contain documents, wiki pages, web links and notes and can be commented upon by other students.

The Community view provides a description of all members of the student group, explicating for each member: the tasks and content items created and responsibilities 
allocated. The community view thus offers insights into the interactions between the personal and social level of the groups' activities.

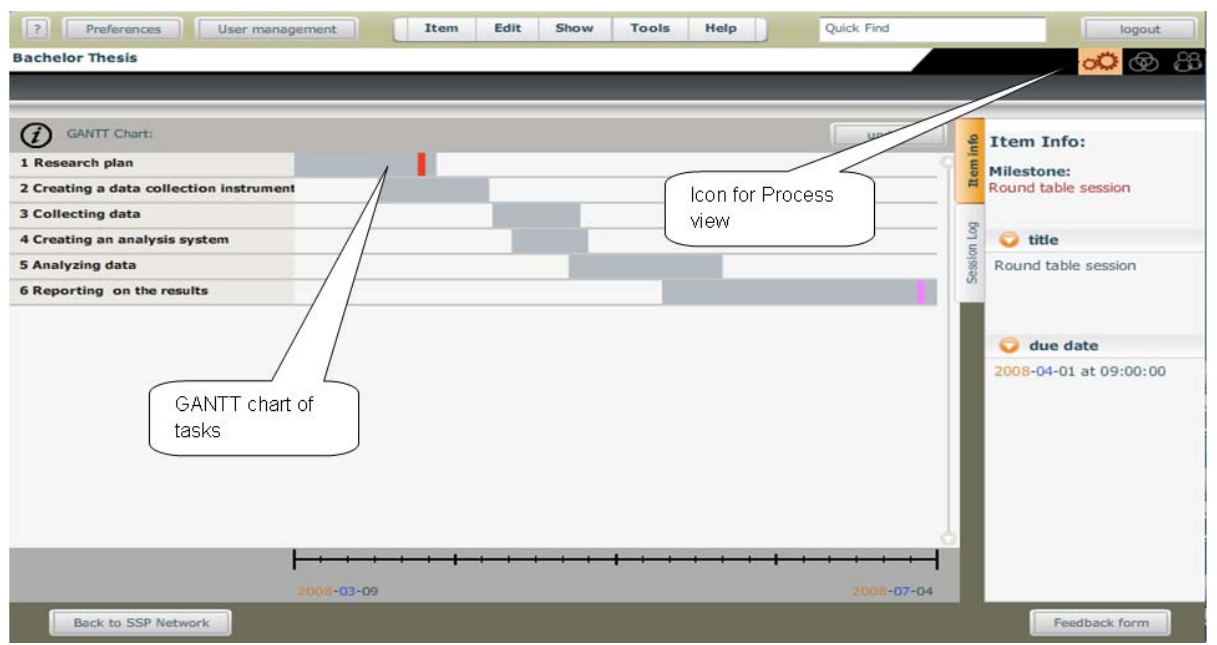

Fig. 2. Process view

To demonstrate how the KP-environment can support trialogical practices we will provide a brief outline of students' practices during the Bachelor thesis scenario below. This is a generic description of the chronology of these practices and of how KP-environment offers support but the sequence and nature of the practices may vary depending on the topic and type of research each student group will be conducting. In addition, practices can be recurrent meaning that they can be revisited during the project depending on the necessity identified by the group members. All the research and other project activities described in the outline are supposed to be performed in collaboration and can be supported by KP-environment.

\section{Project Initiation}

The project initiation takes place at the beginning of the Bachelor thesis course where students are familiarized with the aims of the course. Students have a course manual at their disposal and receive explanations from their tutor about the course set-up. Forming teams and deciding on a research topic is left for students' to chose based on their interests. Student teams write a short essay for their choice, based on arguments found in the scientific research literature and which has to be approved by their tutor before they can continue conducting their research.

The KP-environment community view offers support for students to get acquainted to the other group members and increases awareness of their peers' activities. Groups or individual students who could not make a choice can use the shared space as a mind mapping tool to generate ideas and make a selection of possible topics. Literature sources can be uploaded as content items.

The KP-environment supports students to sketch their ideas directly in their space in the content item view employing the note-editor tool (see Figure 4). The students can compare the versions of the ideas they have produced, since they can be viewed 
in parallel. The note-editor also allows students to synchronously view notes while another student is editing it. In addition, similarly to the other content items inside the shared space, notes can be linked to other relevant material, such as articles students have taken into consideration related to the subject that they are discussing about.

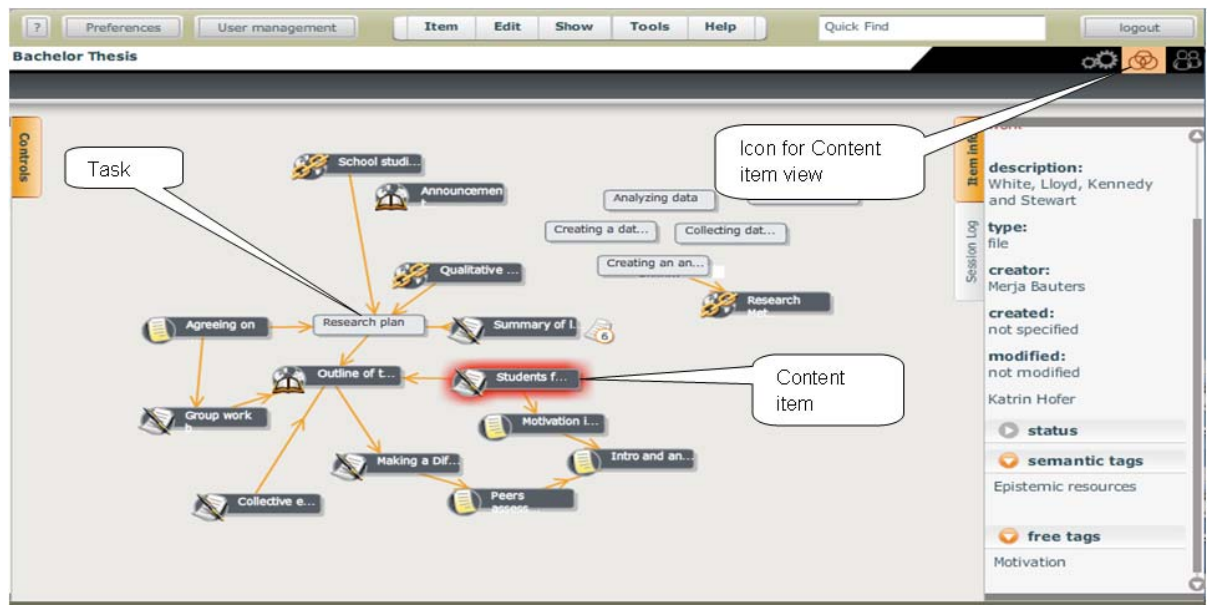

Fig. 3. Content item view

Moreover, Figure 4 shows that students are afforded to rise above previous ideas generated by other groups' members. This gives the chance to rise above the concrete level of brainstorming and commenting. Such discussions, within the context and in relations to their semantic relations, enables collaborative idea generation already from the start of the project. The idea for having these features came from the endusers of previous field trials. Students reported that existing tools did not give them sufficient means for idea generating tasks. Thus, the note-editor tool exemplifies how the KP-environment allows students to collaboratively create, organize and advance their work on knowledge artifacts which increases their sense of ownership and agency over the activities they perform.

The collaborative brainstorming and rising above the previous ideas contributed well to the following design principles: focus on trialogical activity around shared objects (DP1), interaction between personal (DP2) and social level and eliciting (individual and collective) agency (DP5). By enabling contextualized work around shared artifacts (i.e., the notes) (DP1), by allowing individual contributions and idea development allowing others to view, share and contribute into it (DP2) and by engaging the group to collaboratively reflect on which aspects of the provided ideas they have to develop further and investigate (DP5).

\section{Preparing the Research}

The second phase in conducting the project is that students are going to plan and design their research activities. This phase includes writing a research proposal and plan, working out the research problem and elaborating on the theoretical foundations of the research and the sketching of a research design. These artifacts represent the first deliverables of the project. All knowledge necessary for producing these artifacts 
is collected into the shared space in the form of notes and various kinds of documents (i.e. content items) that can be added, re-arranged, tagged and linked with each other (see Figures 2 and 5). In case teams work together on a common research topic, each team will present their approach to the specific sub-topic they are investigating.

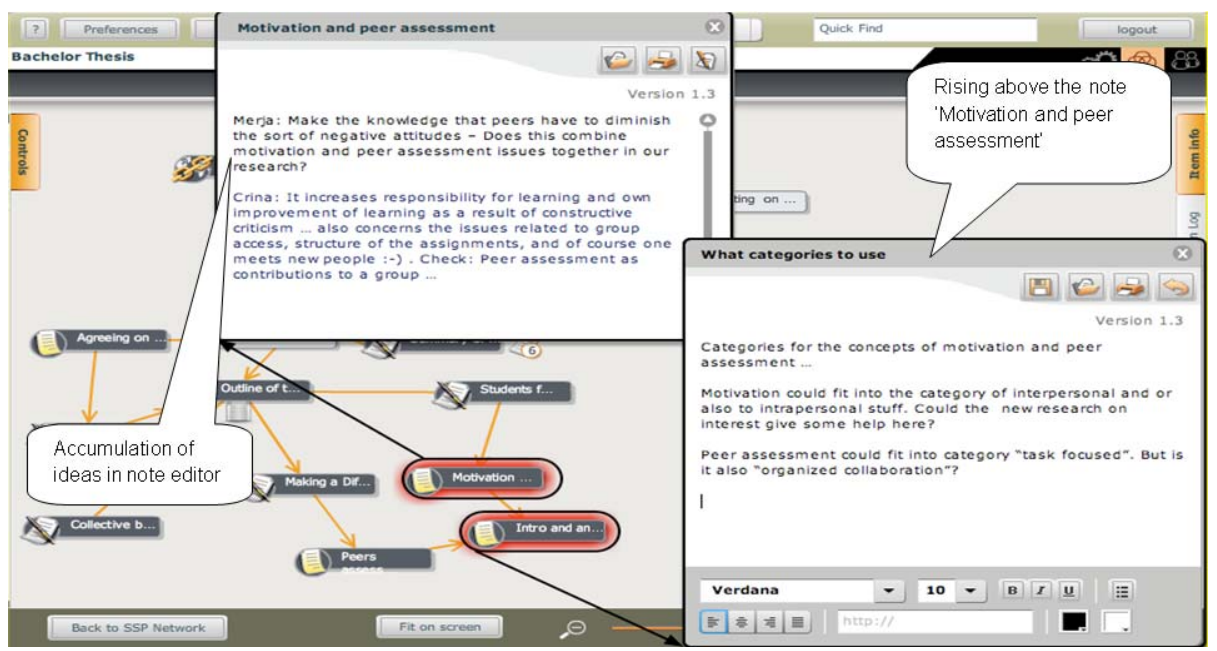

Fig. 4. Two notes that are uploaded in the Content item view. The note at the lower right is in the editing mode and rises above (i.e. categorizes) the note previously generated

As shown in Figure 2, the KP-environment supports planning activities of the student groups since it allows them to create a GANTT chart. This chart enables students to define the tasks to be performed, the timeline associated to the tasks and the responsibilities associated with the particular tasks. Student groups, for instance, produce a research plan, a draft paper (their research report) and a final paper, which must be presented to the other groups and their tutor at a specified date. These critical artifacts can be indicated in the GANTT as milestones. Especially the functionalities of the content item view within the KP-environment provide the main support in this phase of the student groups' project.

Figure 5 presents the commenting tool and shows threads of comments that the particular shared object has promoted. In this case the shared artifact involves a summary of a particular article which receives appraisal of the other students to integrate this artifact in the writing of their final research report. The KP-environment thus supports the commenting of artifacts which enables students to review their work critically and to plan and organize their activities accordingly. The semantic tagging of the shared artifacts helps students to organize the material employing the semantics they assign to the content of the course. As such the KP-environment emphasizes both reflection on content-related work as well as supports the management of collaborative work.

The KP-environment allows the tutor to review the student groups' shared spaces so that he/ she can provide feedback on the artifacts that were created by them. The feedback received from the tutor and from possible other student groups will be used by each team for adjusting and improving the provisional research plans for instance. 


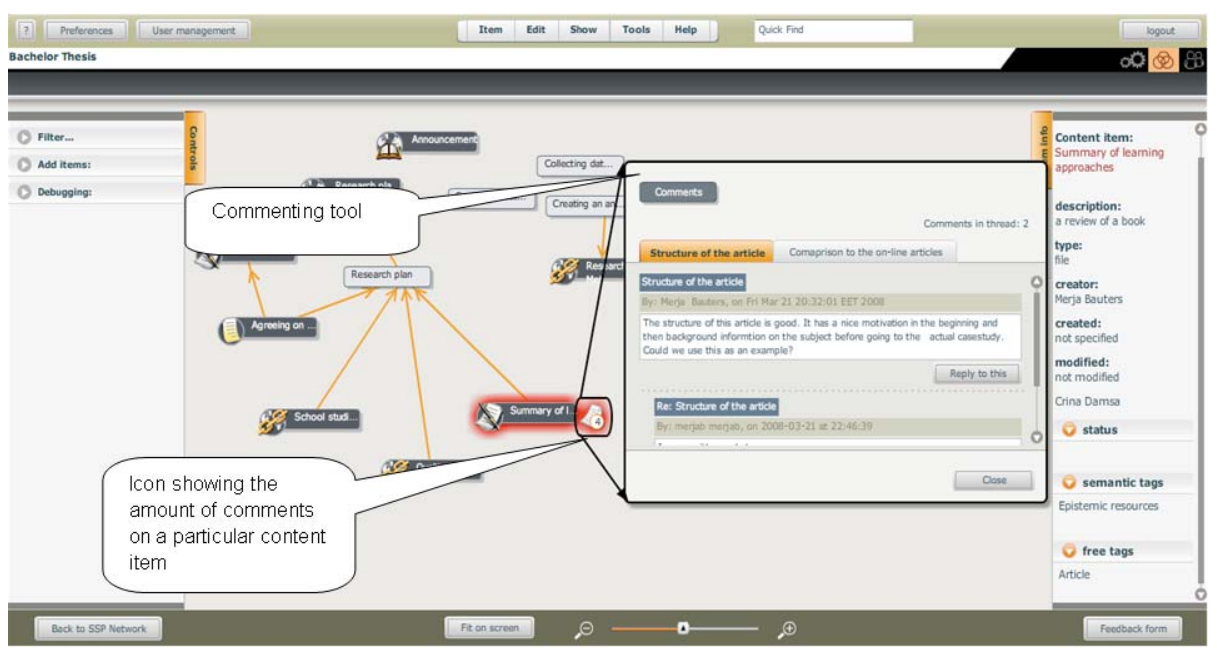

Fig. 5. Commenting on a content item in the KP-environment. As the above screenshot show $\mathrm{s}$ there can be more threads connected to one shared artifact allowing flexible discussion

\section{Conducting the research}

In this phase of the Bachelor thesis scenario, student groups perform activities necessary for the execution of their research. A number of artifacts will be delivered by students, such as data collection instruments, methods for data analysis, data analyses and interpretation reports. For initiating data collection activities a research instrument is needed, therefore brainstorming between the members of the group is important. In addition, students have to think about the design of their research, the nature of the empirical data they require and the ways in which they are going to analyze this data. This also means that the groups have to manage the logistics of conducting their research, which amongst others involves establishing contact with their research participants, collecting some general background information about their participants and the institution they conduct the research. Moreover, students have to plan and elaborate on the nature of their empirical endeavors and the ways in which they are going to extract and analyze data. The KP-environment supports these practices by means of functionalities offered by the GANTT chart in the process view (see Figure 2) and creation, tagging and commenting on content items (see Figures 3, 4 and 5).

At this stage teams also must work on the writing of a provisional research report. This means that they can make intensive use of the note editing tool (see Figure 4). In addition, the KP-environment also affords the student group can to write collaboratively in a Wiki (see Figure 6). The Wiki can be created as content item in the content item view and offers the possibility to write in the same document. The progress and changes made to the document are visible to all group members. 


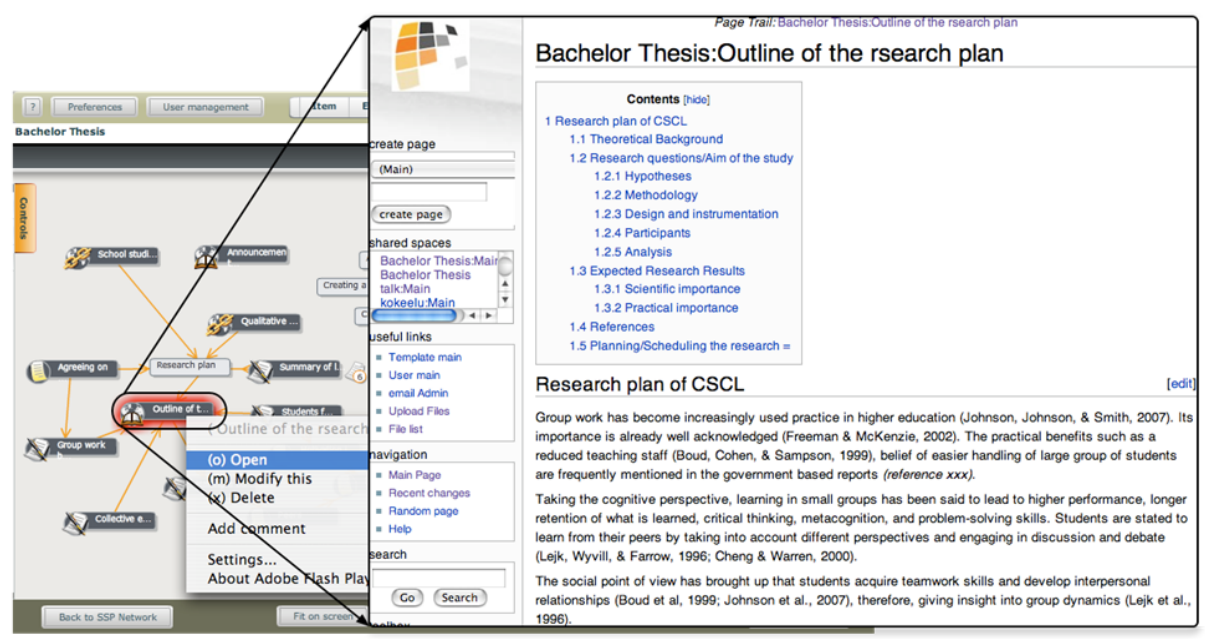

Fig. 6. Content item consisting of a Wiki which outlines a research plan of a particular group

\section{Delivery}

The delivery phase is dominated by the activities for writing the final research report. All the artifacts created by the student group serve to feed into the final research report. The tools offered in the KP-environment enable students to view all the artifacts produced, how they relate to each other, their semantics and their creation process. The research report is iteratively collaboratively revised and commented upon in the student groups' shared space. The collaborative writing activities prevail in this project phase which means that the collaboration tools in the KP-environment such as the note editor, the Wiki, the commenting and semantic tagging tools are important during this period. The Gantt chart in the process view of the KPenvironment provides an insight in the advancement of the groups' collaborative object-related work. In the end, the report is published and the tutor and other groups can give evaluative feedback on the artifacts produced and on the main object delivered.

\section{Conclusion and Discussion}

To design learning technologies in such a way that competencies, which are essential to be able to productively participate within this production oriented society, become more central necessitates for a reconceptualization of the way in which learners organize their knowledge work. The emphasis in designing technology should not merely focus on individual knowledge acquisition or on social interaction, but more on the way in which learners collaboratively create and advance knowledge objects [6]. In this paper we presented the KP-environment which is a virtual collaboration space which attempts to afford its users to manage, organize and elaborate on the artifacts they create during a trialogical learning or working process. The considerations that were taken into account in the design of the KP-environment for 
supporting these practices were described as well as its application for a concrete scenario in which students collaboratively construe a research report.

Although the KP-environment is part of the whole trialogical pedagogical context, it fosters those practices deemed essential in knowledge creation. This means that it focuses on the sustained trialogical activity around shared artifacts through offering flexible tools which support: a) elaboration on content related semantics of the tasks students decide they have to perform, b) communal practices to cross fertilize between different partners in a group, fostering sustained processes of knowledge advancement and move beyond the individual level, and c) management and organization of the groups' practices.

At present KP-environment represents a developmental trail towards supporting trialogical learning, which offers a perspective on preparing students for authentic knowledge management work. Nevertheless, it needs to be noted that the present paper presented the design considerations and the foreseen usage of the KPEnvironment for particular practices in a specified pedagogical scenario that has to be implemented yet. In order to validate the KP-environment, empirical research is needed in which its educational significance is tested and evaluated.

\section{References}

1. Bereiter, C.: Education and mind in the knowledge age. Lawrence Erlbaum, Hillsdale NJ (2002)

2. Engeström, Y.: Activity theory and individual and social transformation. In: Engeström, Y., Miettinen, R., \& Punamäki R.-L. (eds.) Perspectives on activity theory, pp. 19--38. Cambridge University Press, Cambridge MA (1999)

3. Latour, B.: Pandora's hope. Harvard University Press, Cambridge MA (1999)

4. Andriessen, J.E.B.: Arguing to learn. In: Sawyer, R.K. (Ed.) The Cambridge handbook of the learning sciences, pp. 443--460. Cambridge University Press, New York (2006)

5. Sfard, A.: On two metaphors for learning and the dangers of choosing just one. Educational Researcher 27(2), 4--13 (1998)

6. Paavola, S. \& Hakkarainen, K.: The Knowledge Creation Metaphor - An Emergent Epistemological Approach to Learning. Science \& Education. 14(6), 535--557 (2005)

7. Engeström, Y.: Learning by expanding. Orienta - Konsultit Oy, Helsinki (1989)

8. Star, S. L.: The structure of ill-structured solutions: Boundary objects ands heterogeneous distributed problem solving. In L Glasser, M. N. Huhns (eds.) Distributed artificial intelligence, vol. II, pp. 37--54. Pitman, London (1989)

9. Scardamalia, M.: Collective cognitive responsibility for the advancement of knowledge. In B. Smith (Ed.) Liberal Education in a Knowledge Society, pp. 67-98. Open Court, Chicago (2002)

10.Bell, P., Hoadley, C. M., \& Linn, M. C.: Design-based research. In M. C. Linn, E. A. Davis, \& P. Bell (eds.) Internet environments for science education, pp. 73--88. Lawrence Erlbaum Associates, Mahwah New Jersey (2004) 